\title{
Gene-to-Gene Network Analysis of the Mediation of Plant Innate Immunity by the Eliciting Plant Response-Like 1 (Epl1) Elicitor of Trichoderma formosa
}

\author{
Chi-Hua Cheng, ${ }^{1}$ Bing-Nan Shen, ${ }^{2}$ Qian-Wen Shang, ${ }^{2}$ Li-Yu Daisy Liu, ${ }^{3}$ Kou-Cheng Peng, ${ }^{4}$ \\ Yan-Huey Chen, ${ }^{2}$ Fang-Fang Chen, ${ }^{2}$ Sin-Fen Hu, ${ }^{2}$ Yu-Tai Wang, ${ }^{5}$ Hao-Ching Wang, ${ }^{6} \mathrm{Hsin}-\mathrm{Yi} \mathrm{Wu},{ }^{7}$ \\ Chaur-Tsuen Lo, ${ }^{1,+}$ and Shih-Shun Lin ${ }^{2,5,8,9,+}$ \\ ${ }^{1}$ Department of Biotechnology, National Formosa University, Yulin, Taiwan; ${ }^{2}$ Institute of Biotechnology, National Taiwan \\ University, Taipei, Taiwan; ${ }^{3}$ Department of Agronomy, National Taiwan University; ${ }^{4}$ Institute of Biotechnology, National Dong- \\ Hwa University, Hualien, Taiwan; ${ }^{5}$ National Center for High-Performance Computing, National Applied Research Laboratories, \\ Hsinchu, Taiwan; ${ }^{6}$ Graduate Institute of Translational Medicine, College of Medical Science and Technology, Taipei Medical \\ University, Taipei, Taiwan; ${ }^{7}$ Instrumentation Center, National Taiwan University; ${ }^{8}$ Agricultural Biotechnology Research Center, \\ Academia Sinica, Taipei, Taiwan; and ${ }^{9}$ Center of Biotechnology, National Taiwan University
}

Accepted 9 February 2018.

\begin{abstract}
A new clade, Trichoderma formosa, secretes eliciting plant responselike 1 (Epl1), a small peptide elicitor that stimulates plant immunity. Nicotiana benthamiana pretreated with Epl1 for 3 days developed immunity against Tomato mosaic virus (ToMV) infection. The transcriptome profiles of $T$. formosa and $N$. benthamiana were obtained by deep sequencing; the transcript of Epll is $736 \mathrm{nt}$ in length and encodes a 12-kDa peptide. Identifying critical genes in Epl1-mediated immunity was challenging due to high similarity between the transcriptome expression profiles of Epl1-treated and ToMV-infected $N$. benthamiana samples. Therefore, an efficient bioinformatics data mining approach was used for high-throughput transcriptomic assays in this study. We integrated gene-to-gene network analysis into the ContigViews transcriptome database, and genes related to jasmonic acid and ethylene signaling, salicylic acid signaling, leucine-rich repeats, transcription factors, and histone variants were hubs in the gene-to-gene networks. In this study, the Epl1 of T. formosa triggers plant immunity against various pathogen infections. Moreover, we demonstrated that high-throughput data mining and gene-to-gene network analysis can be used to
\end{abstract}

${ }^{\dagger}$ Corresponding authors: S.-S. Lin; E-mail: linss01@ntu.edu.tw and C.-T. Lo; E-mail: ctlo@nfu.edu.tw

Funding: This work was supported by the Ministry of Science and Technology NSC99-2324-B-150-002 and NSC 101-2324-B-150-002 to ChaurTsuen Lo and MOST 106-2321-B-002-008- to Shih-Shun Lin.

The raw transcriptome reads reported in this paper are available in the National Center for Biotechnology Information Short Read Archive under accession numbers SRR5500248 (healthy Nicotiana benthamiana), SRR5500783 (Tomato mosaic virus [ToMV]-infected N. benthamiana), SRR5500769 (Epl1-treated N. benthamiana), and SRR5500770 (Ep11+ ToMV-infected $N$. benthamiana). The GenBank accession numbers are MF039479 (Epll) and MF043189 (RPB2). The transcriptome database of $N$. benthamiana is available in the ContigViews database.

*The $\boldsymbol{e}$-Xtra logo stands for "electronic extra" and indicates that three supplementary figures and two supplementary tables are published online.

๑) 2018 The American Phytopathological Society identify critical candidate genes for further studies on the mechanisms of plant immunity.

To study molecular plant and microbe interactions, researchers need to identify critical genes involved in interesting phenomena, such as the development of symptoms or resistant phenotypes. However, most of the important problems in plant disease occur in crops that might not have genomic or transcriptomic information available, the so-called nonmodel organisms. Currently, nextgeneration sequencing (NGS) is widely applied to study the whole-transcriptome profiles of model and nonmodel organisms and to understand gene expression from a genome-wide perspective (Gao et al. 2014; Kim et al. 2014; Liu et al. 2014; Castro et al. 2015; Lin et al. 2015). Although NGS can easily generate contigs for genomes or transcriptomes, the bottleneck is how to efficiently analyze these high-throughput genomic profiles.

The ContigViews transcriptome database was designed for analyzing the transcriptome profiles of nonmodel organisms. Liu et al. (2014) first used the ContigViews system to study the transcriptome of Catharanthus roseus (a nonmodel organism). The system has automatic functions for open reading frame (ORF) annotation that efficiently identify genes and noncoding transcripts from de novo assembled contigs (Liu et al. 2014). That study reported approximately $88 \%$ identity between NGS and microarray transcript expression data, suggesting that NGS is highly accurate.

Based on a previous study, the whole-transcriptome profiles of $C$. roseus provide gene sequences and information regarding differentially expressed genes (DEGs) under different treatments or conditions (Liu et al. 2014). However, approximately 3,000 DEGs are revealed in each comparison, and it is difficult to determine which of these many DEGs are critical genes that can be used as targets for further study (Liu et al. 2014). A gene-to-gene network is typically constructed based on the similarities of target gene expression profiles over time or under different treatment conditions (Liu 2015). The resulting networks can assist in contextualizing the intricate expression profiles at the system level and enhance our understanding of relevant biological processes at the molecular level (Serin et al. 
2016). The applications of the inferred gene expression networks in plants include identification of functional gene modules (Bassel et al. 2011; Mutwil et al. 2010), the functional annotation of genes (Childs et al. 2011; Vandepoele et al. 2009), the identification of causal genes in targeted biological processes (Chan et al. 2011; Jiménez-Gómez et al. 2010; Ransbotyn et al. 2015) and studies on evolution (Ficklin and Feltus 2011; Mutwil et al. 2011). Liu et al. (2014) established a gene-to-gene network based on the transcriptome profiles of various healthy flower tissues and flower tissues infected with peanut witches' broom phytoplasma to identify several critical genes in $C$. roseus plants that might be involved in leafy flower symptom development. Thus, the use of gene-to-gene networks can be considered a 'big data mining' approach.

Pathogen-associated molecular patterns (PAMPs) and microbial-associated molecular patterns (MAMPs), which are generated by pathogens or nonpathogenic microorganisms, induce primary PAMP- or MAMP-triggered immunity (PTI/MTI) to counteract invading organisms. Pathogen effectors are generated to suppress PTI/MTI. However, various resistance genes ( $R$ genes) have evolved in plants to recognize these effectors and promote effector-triggered immunity (ETI) (Chisholm et al. 2006). Plant ETI provides systemic resistance by sensing pathogenic effectors to trigger induced systemic resistance (ISR) or systemic acquired resistance (SAR), which constitute different layers of innate immune responses (Chisholm et al. 2006; Durrant and Dong 2004; Ton et al. 2002).

Trichoderma spp. are used as biocontrol agents against a variety of plant pathogens; these fungi secrete cerato-platanin elicitors (approximately $12 \mathrm{kDa}$ ), including eliciting plant response-like 1 (Epl1) and small protein-1 (Sm1), both of which have been shown to trigger MTI against pathogens (Kottb et al. 2015; Salas-Marina et al. 2015; Seidl et al. 2006). Epl1 and Sm1, which have highly conserved amino acid sequences, induce the jasmonic acid (JA) and ethylene (ET) signaling pathways to trigger ISR. However, overlapping ISR- and SAR-related genes have been detected in plants treated with Epl1 and Sm1, which suggests that these elicitors function synergistically in both systems of innate immunity (Martínez-Medina et al. 2013; SalasMarina et al. 2015). To date, the underlying molecular mechanism has not been fully elucidated.

In this study, we identified Trichoderma formosa, which is a new clade of Trichoderma spp. The identified secreted Epl1 can trigger plant innate immunity against different pathogen infections. Moreover, gene-to-gene network analysis was incorporated into the ContigViews NGS transcriptome platform, and we identified several critical genes that might be involved in T. formosa Epl1-induced innate immunity.

\section{RESULTS}

Trichoderma formosa is a new clade of Trichoderma spp.

T. formosa was isolated in Changhua County, Taiwan in 1997. A phylogenetic tree based on RPB2 (RNA polymerase II subunit $B$ ) indicates that $T$. formosa is genetically close to Trichoderma atroviride and Trichoderma paratrociride (Fig. 1A). Based on the phylogenetic tree, T. formosa can be classified into a new clade of Trichoderma spp. (Fig. 1A; Supplementary Fig. S1). T. formosa exhibits broad, regular, tree-like peripheral conidiophores and dense central clusters (Fig. 1B).

\section{Size exclusion chromatography of $\boldsymbol{T}$. formosa culture medium.}

T. formosa secretes an elicitor protein into the culture medium. We collected the medium and purified the elicitor protein, using fast protein liquid chromatography (FPLC) with a HiPrep 16/60 Sephacryl S-100HR column (Fig. 1C, panel i).
We established three zones based on the UV values of the fractions (zone A, zone B, and zone C) (Fig. 1C, panel i). The fractions were analyzed by 12 or $15 \%$ polyacrylamide gel electrophoresis (PAGE) (Fig. 1C, panels ii and iii). Several bands were obtained via $12 \%$ PAGE (Fig. 1C, panel ii), whereas 12- and 24-kDa bands were detected via 15\% PAGE (Fig. 1C, panel iii). In $15 \%$ PAGE, zone $\mathrm{A}$ represents the $12-\mathrm{kDa}$ bands, zone $\mathrm{B}$ represents the 12 - and $24-\mathrm{kDa}$ bands, and zone $\mathrm{C}$ represents the 24-kDa bands. The 12- and 24-kDa bands were further analyzed by liquid chromatography-tandem mass spectrometry (LC-MS/MS) after in-gel digestion. The results indicated that the 24- and 12-kDa bands are Epl1 (Fig. 1D; Supplementary Table S1). Therefore, we assume that the 24-kDa band is Epl1 in dimer form and that the $12 \mathrm{kDa}$ band is the Epl1 monomer (Fig. 1C). Why might the dimeric form of Epl1 migrate more slowly than the monomeric form? We assume that dimers may form compact structures, making them smaller than the monomers. Notably, a mixture of dimeric and monomeric Epl1 was used for subsequent experiments. According to the report of Seidl et al. (2006), the N-terminal 18 amino acids compose a signal peptide (Fig. 1D, red box) that might allow Epl1 to be directly secreted into the culture medium.

\section{Epl1 triggers plant resistance to various pathogens.}

To evaluate whether Epl1 triggers resistance to a plant virus, we treated Nicotiana benthamiana plants with $10 \mathrm{nM}$ Epl1 for 3 days and, then, inoculated the plants with Tomato mosaic virus (ToMV). At 7 days postinoculation, no symptoms were observed on the Epl1-treated leaves (Fig. 1E, panel iv); however, symptoms of necrosis were observed on the nonEpl1-protected leaves (Fig. 1E, panel ii). Furthermore, enzymelinked immunosorbent assay failed to detect ToMV infection on the Epl1-protected N. benthamiana plants, suggesting ToMV resistance (Fig. 1F). Additionally, sliced carrot tubers were treated with $1 \mathrm{nM}$ Epl1 and were infected with Pectobacterium carotovorum. No lesions or lysis caused by the $P$. carotovorum infection were observed on the Epl1-treated tubers (Fig. 1G, left panel). In contrast, a mock-inoculated sample exhibited soft rot symptoms 3 days after P. carotovorum infection (Fig. 1G, right panel). Moreover, there was no difference in the resistance to $P$. carotovorum based on the number of Epl1-protected days or the Epl1 concentration (Fig. 1H). The 1-day Epl1 treatment was just as efficient as the 3-day treatment in promoting resistance (Fig. 1H). We then evaluated Epl1-mediated resistance to Alternaria brassicicola infection in Brassica rapa subsp. chinensis plants. B. rapa subsp. chinensis plants were treated with $10 \mathrm{nM}$ Epl1 and showed resistance to A. brassicicola, whereas necrotic lesions were observed on mock-inoculated leaves 6 days after inoculation (Fig. 1I). The same resistance was observed over various days after Epl1 treatment (Fig. 1J). These results indicated that Epl1 treatment triggered the plant immune systems of carrot and $B$. rapa subsp. chinensis against $P$. carotovorum and A. brassicicola, respectively.

\section{Identification of the Epl1 gene in T. formosa.}

We analyzed the transcriptome of T. formosa to obtain gene sequence information. We obtained 4,892 complete genes (28.9\%), 4,549 partial genes (26.9\%), and 7,499 undefined contigs (44.4\%) (Fig. 2A). The Epll transcript is $736 \mathrm{nt}$ in length, including an ORF (417 nt) with $5^{\prime}$ - and $3^{\prime}$-untranslated regions (Fig. 2B). There is an 18-amino acid signal peptide sequence (Fig. 2B, red box) located at the N-terminus of Epl1. We cloned the Epll gene from genomic DNA and found one intron at nt 62 (from the first ATG) (Supplementary Fig. S2). In addition, the ORF sequence of the genomic DNA was $100 \%$ identical to the Epll transcript, suggesting that the de novo transcriptome assembly is highly accurate. A phylogenetic tree 
of the Epl1 and Sm1 proteins from various Trichoderma spp. revealed that Epl1 and Sm1 could be divided into two groups (Fig. 2C). Each group could then be divided into three subgroups (Fig. 2C). The Epl1 proteins in T. formosa and T. gamsii T6085 were classified in the G1 subgroup (Fig. 2C).

The crystal structure of $T$. virens $\mathrm{Sm} 1$, which had been previously determined (Protein Data Bank accession number 3M3G), shows that Sm1 contains seven alpha helices and seven beta strands. A zinc bromide located on the Sm1 surface might help maintain one or both the structure and the function of the protein (Fig. 2D). Figure 2E presents an alignment of the Epl1 and Sm1 amino acid sequences. Based on the Sm1 protein structure and the alignment, Sm1 and Epl1 differ by 11 amino acids (Fig. 2E, red arrowheads) in the unstructured regions and by 10 amino acids (Fig. 2E, black arrowheads) in the structured regions. Moreover, two amino acids are different among the subgroups (Fig. 2E). In the glycosylated region (Fig. 2E, red box), there is a 1 amino acid difference between the two elicitors, Epl1 has Ala30 but Sm1 has Gly30 (Fig. 2E). This result provides information for further studies on the differences between Epl1 and Sm1.

\section{Whole-transcriptome analysis of Epl1-treated $N$. benthamiana.}

Transcriptome profiles were obtained from various $N$. benthamiana samples, including mock-inoculated healthy plants (Mock), ToMV-infected plants (ToMV), Epl1-treated plants (Epl1), and Epl1-treated and ToMV-infected plants (Epl1+ToMV) (Fig. 3A). The Epl1+ToMV sample refers to $N$. benthamiana treated with Epl1 for 3 days and then challenged with ToMV. Each sample produced approximately 28 million reads (Fig. 3A). After de novo assembly by mixing all reads from the four samples, 128,063 contigs were generated, and the $\mathrm{N}_{50}$ was $779 \mathrm{nt}$ (Fig. 3A). After the ORF annotation, there were 40,243 genes and 87,820 undefined transcripts (Fig. 3A and B). Among the 40,243 genes, 5\% of the contigs $(6,435$ genes) represented complete genes, and $26.4 \%$ of the contigs $(33,808$ genes) represented partial genes (Fig. 3B). The other $68 \%$ of the contigs were classified as undefined transcripts, which might be noncoding RNAs (Fig. 3B). The amino acids of $N$. benthamiana and Arabidopsis thaliana were 55\% similar (Fig. 3C). Notably, the transcriptome database is available on ContigViews.

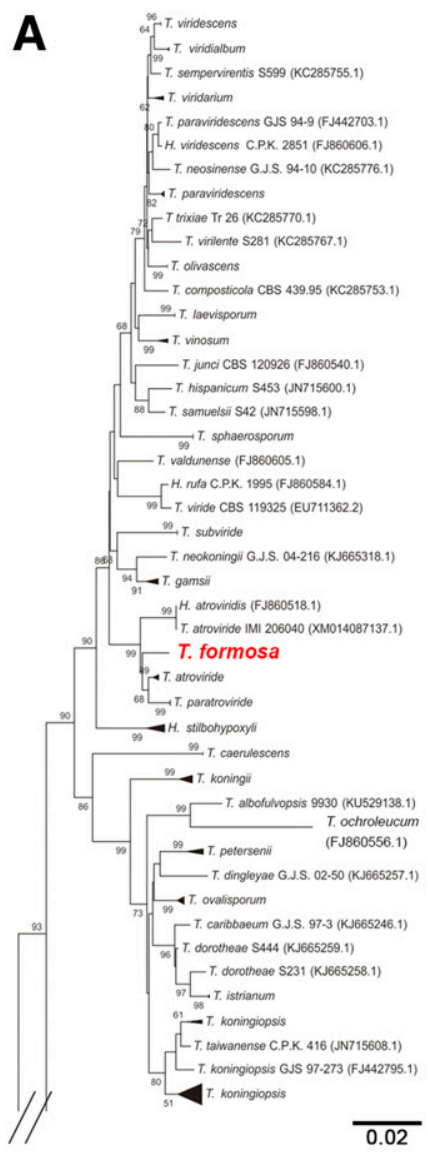

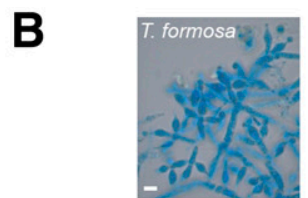

D \begin{tabular}{l} 
MQFSSLFKLALFTAAVSADTVSYDTGYDDASR \\
\hline SLTVVSCSDGANGLITRYHWQTQGQIPRFPYI \\
GGVQAVAGWNSPSCGTCWKLTYSGKTIYVLA \\
VDHSAAGFNIGLDAMNALTNGNAVQYGRVDA
\end{tabular} TASQVAVKNCGL'

E

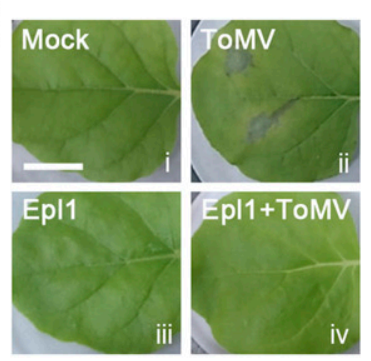

F

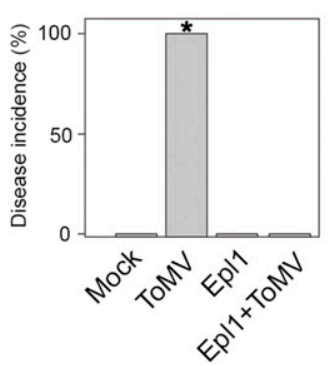

C i

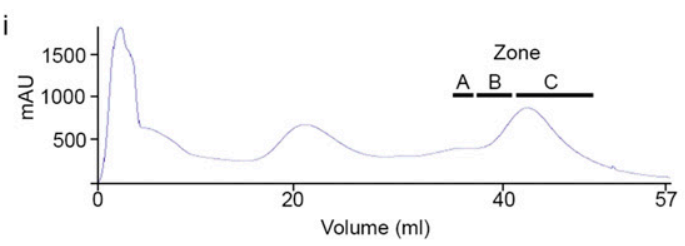

ii

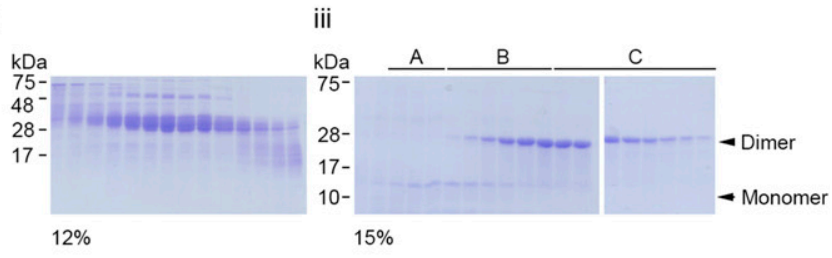

G

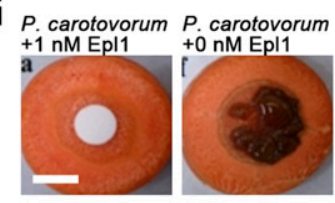

H

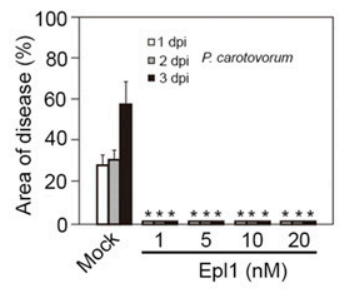

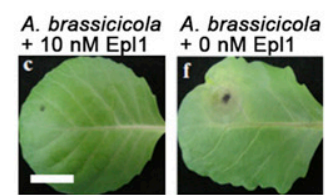

J

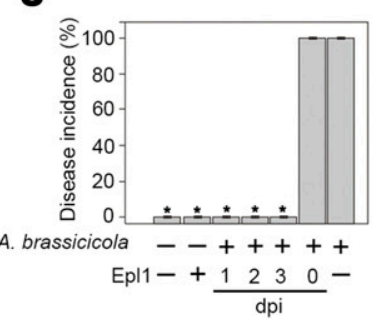

Fig. 1. Characterization of Trichoderma formosa and the Epl1 effector for triggering plant defense responses. A, Phylogenetic tree of RPB2 genes in Trichoderma spp. Bar $=5 \mu \mathrm{m}$. B, Morphology of T. formosa. C, Monomers and dimers of purified Epl1. D, The matching peptides in the Epl1 amino acid sequence are marked in green and red. E, Evaluation of Epll-mediated resistance in Nicotiana benthamiana: i, mock-inoculated healthy plants (Mock); ii, Tomato mosaic virus (ToMV)-infected leaf; iii, Epl1-treated leaf; iv Epl1-protected leaf challenged with ToMV. Photographs taken at 7 days postinoculation (dpi). Bar $=5 \mathrm{~cm}$. F, Evaluation of ToMV disease incidence in Nicotiana benthamiana pretreated with or without Epl1. Error bars represent standard error $(n=$ 3). The disease incidence was significantly different from that in the control in each sample, based on Student's $t$ test. One asterisk (*) indicates $P<0.05$. G, Epl1 protects carrot against Pectobacterium carotovorum infection (left panel). P. carotovorum was infected on the untreated carrot (right panel). Bar $=5 \mathrm{~cm}$. $\mathbf{H}$, Measurements of $P$. carotovorum-infected area in carrot tissues pretreated with various protected days of Ep11. Error bars represent the standard error $(n=$ 3 ). The percentages of areas that were infected significantly differed from those in $P$. carotovorum in each sample, as indicated by Student's $t$ test. One asterisk $(*)$ indicates $P<0.05$. I, Epl1 triggers protection against Alternaria brassicicola infection in Brassica rapa subsp. chinensis. Bar $=5 \mathrm{~cm} . \mathbf{J}$, Observation of the A. brassicicola disease incidence (\%) on B. rapa subsp. chinensis leaves pretreated with various protected days of Epl1. Error bars represent standard error $(n=3)$. The disease incidence was significantly different from that in the control in each sample, based on Student's $t$ test. One asterisk $(*)$ indicates $P<0.05$. 


\section{Differential expression}

under various treatment conditions.

In this study, 40,243 annotated genes, including complete and partial genes, were selected for a comparison of their expression. The gene expression profiles of the Mock, ToMV, Epl1, and ToMV+Epl1 samples were analyzed using ContigViews. First, we identified 10,930 complete and partial genes. The partial genes were selected based on a $>50 \%$ sequence coverage relative to the Arabidopsis sequence. Second, we compared the gene expression in twofold change in each sample and generated an expression heatmap (Fig. 3D). We compared the heatmap patterns of ToMV to those of Mock (ToMV/Mock), Epl1 to Mock (Epl1/Mock), and Epl1+ToMV to Mock (Epl1+ToMV/Mock) (Fig. 3D). Surprisingly, similar patterns were observed in the ToMV/Mock and Epl1/Mock heatmaps, whereas the pattern of Epl1+ToMV/Mock was mostly the opposite, suggesting that Epl1 may trigger biological responses similar to those triggered by ToMV infection (Fig. 3D). Figure 3E shows the gene expression curves for the Mock, Epl1, ToMV, and Epl1+ToMV samples. Like the heatmaps, the expression patterns from the Epl1 and the ToMV samples are similar and can be divided into two types (Fig. 3E). The genes with a type I expression pattern are up-regulated in both Epl1 and ToMV, whereas genes with a type II expression pattern are down-regulated in both Epl1 and ToMV (Fig. 3E). The red curves in the type I and II patterns revealed genes that were identified from the gene-to-gene network assay (described below).

Although the expression patterns between the Epl1 and ToMV samples were similar, several critical genes involved in immunity might have different patterns between the two samples. For instance, NbPR4 (NBBAC1048), which is a marker gene of the salicylic acid (SA) pathway, was up-regulated in the ToMV sample but down-regulated in the Epll sample (Fig. 3F). Interestingly, the other SA pathway-related genes, e.g., NbCALS1 (NBBAC17244), NbNPR1 (NBBAC15760), NbNPR3 (NBBAC8831), NbNPR4 (NBBAC8017), NbPAL1 (NBBAC5944), and NbEDS1 (NBBAC12129), were up-regulated
A

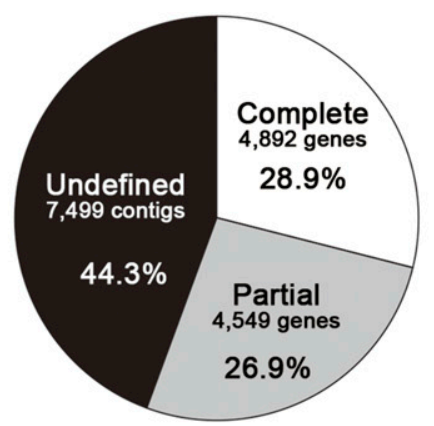

\section{T. formosa transcriptome profile}

B

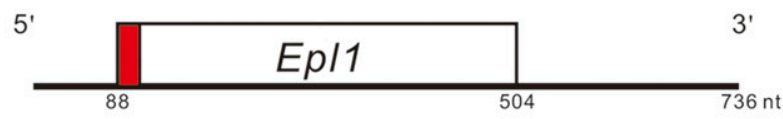

\section{C}

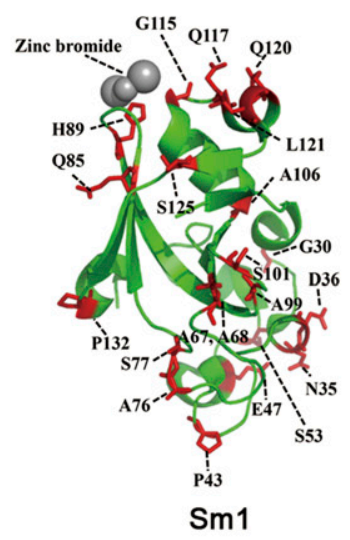

W $\quad$ T

E

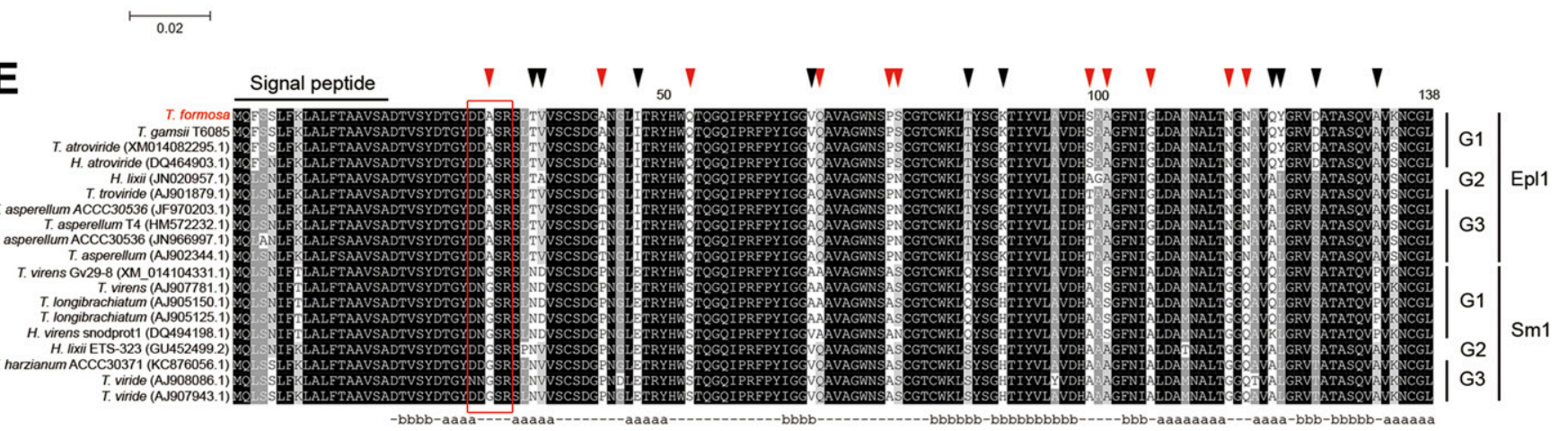

Fig. 2. Characterization of Epl1 in Trichoderma formosa. A, Percentages of complete genes, partial genes, and undefined transcripts in T. formosa. B, Illustration of the Epl1 transcript. A box indicates the open reading frame. The red box indicates signal peptide. C, Phylogenetic tree of Epl1 and Sm1. D, The three-dimensional structure of Sm1 in T. virens. E, Multiple alignments of Epl1 and Sm1 from various Trichoderma spp. The red box indicates the glycosylation site. Red arrowheads indicate different amino acids in the unstructured regions. Black arrowheads indicate different amino acids in the structured regions. 
in the Epl1 and ToMV samples whereas NbJAR1.2 (Jasmonate Resistant 1, NBBAC9520, a JA pathway-related gene) was down-regulated in the ToMV sample but up-regulated in the Epl1 sample (Fig. 3F; Supplementary Figure S3). These data demonstrated that genes related to JA and SA signaling were both induced in the Epl1 sample (Fig. 3F).

\section{Gene-to-gene network.}

To further investigate the mechanism of Epl1-mediated innate immunity, we conducted a gene-to-gene network analysis using the ContigViews-Network to elucidate the gene-to-gene connections in the Epl1/Mock and ToMV/Mock networks. In brief, the genes for network construction were selected
A

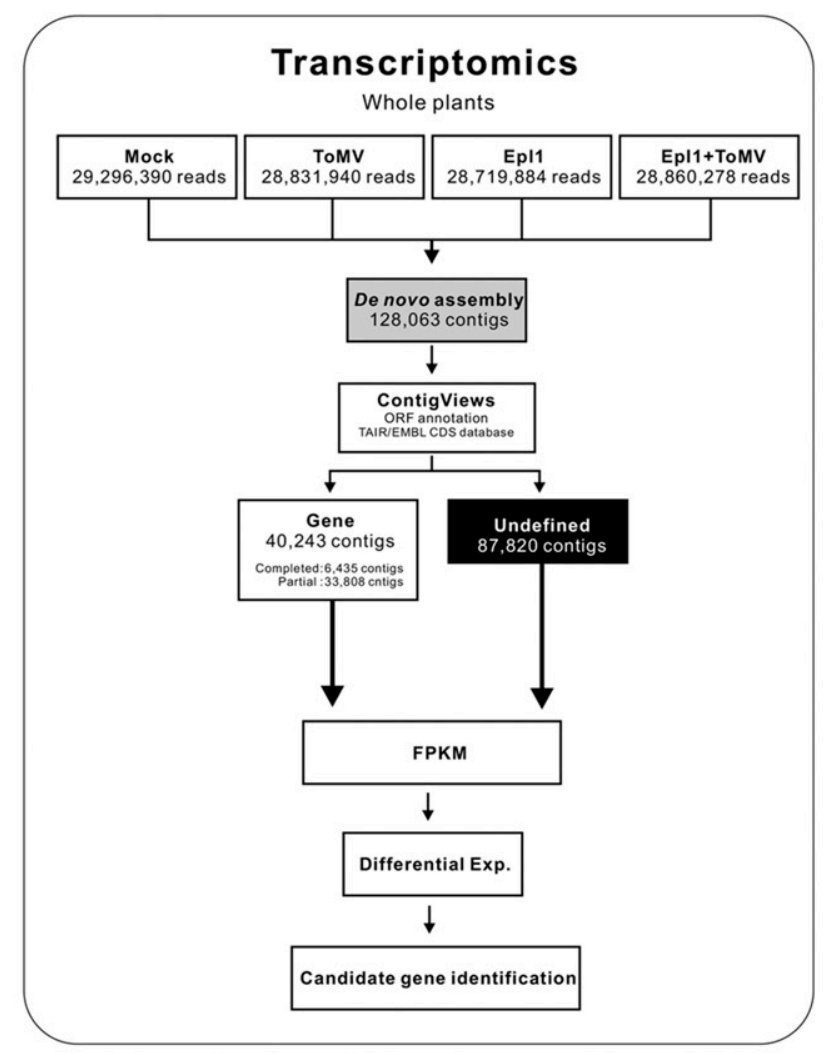

$\mathbf{E}$
B

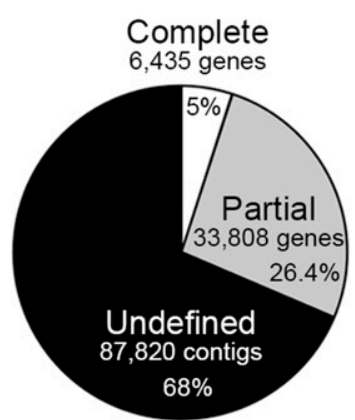

$N$. benthamiana transcriptome

C

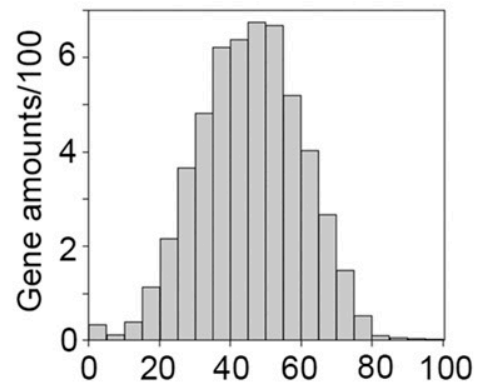

Similarity of amino acids (\%)
D

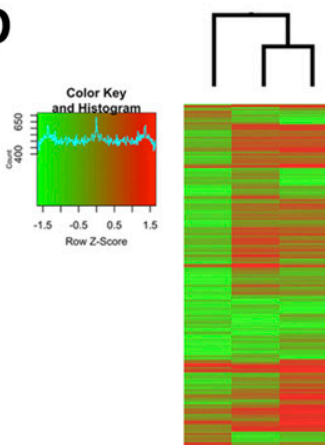

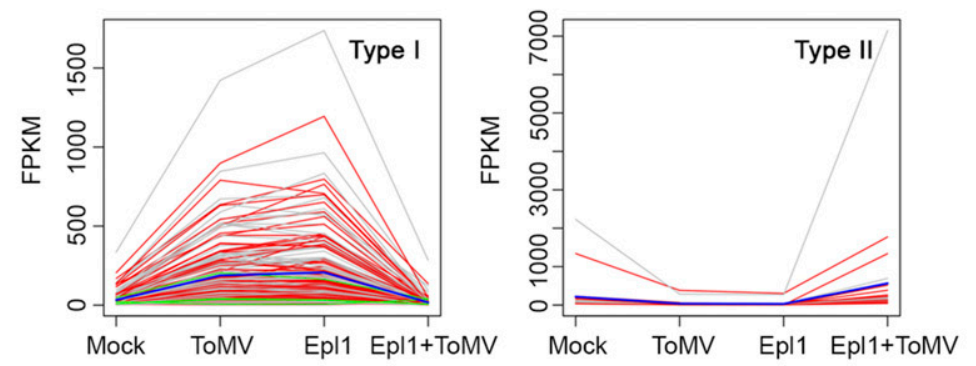

F i
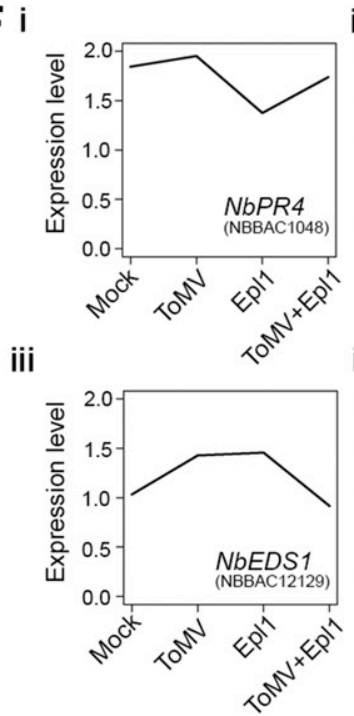

ii
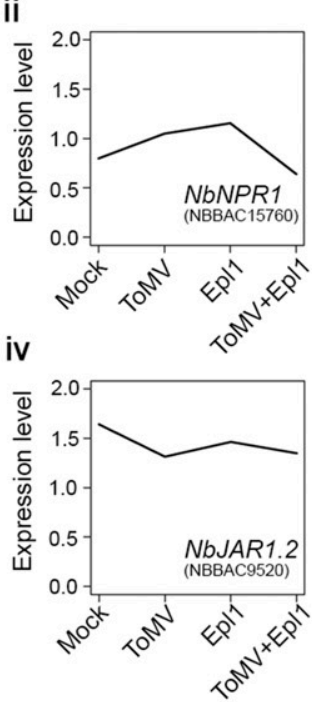

Fig. 3. Whole-transcriptome next-generation sequencing analysis workflow. A, Whole-transcriptome analysis pipeline. Healthy Nicotiana benthamiana plants (Mock), Tomato mosaic virus (ToMV)-infected N. benthamiana plants (ToMV), N. benthamiana plants pretreated with Epl1 for 1 day (Epl1), and ToMVinfected Epl1-pretreated $N$. benthamiana plants (Epl1+ToMV) were selected for whole-transcriptome analysis using deep sequencing and measured as fragments per kilobase of transcript per million mapped reads (FPKM), using gene ontology (GO), Kyoto Encyclopedia of Genes and Genomes (KEGG), and InterPro domain databases. The 128,063 de novo assembled N. benthamiana contig sequences were compared with The Arabidopsis Information Resource and European Molecular Biology Laboratory coding sequence (CDS) databases through BLAST for open reading frame annotation. B, Percentages of complete genes, partial genes, and undefined contigs in N. benthamiana. C, Amino acid similarity in the CDSs of Arabidopsis and N. benthamiana. D, Epl1+ ToMV/Mock represents the expression pattern of the Epl1+ToMV sample relative to that of the Mock sample; "Epl1/Mock" represents the expression patterns of the Epl1 sample relative to that of the Mock sample; ToMV/Mock represents the expression pattern of the ToMV sample relative to that of the Mock sample. $\mathbf{E}$, Two types of gene expression patterns in four samples. The red curves indicate the genes highlighted in the gene-to-gene network maps. The blue curve indicates the average gene expression level in the same pattern. F, The gene expression patterns of $N b P R 4$ (NBBAC1048), NbNPR1 (NBBAC15760), NbEDS1 (NBBAC12129), and NbJAR1.2 (NBBAC9520). The expression levels are FPKM after being $\log _{2}$ transformed twice. 
according to their expression levels. The fragments per kilobase of transcript per million mapped reads (FPKM) values of these genes were $\log _{2}$-transformed twice to ensure their normality for further correlation calculations. The genes with transformed FPKM values less than 1.14 were discarded. Then, the correlation between the transformed FPKM values of any pair of genes was calculated to determine the level of association between the two genes. Network maps were generated, using ContigViews, based on gene-to-gene correlations.

The genes present in the Epl1/Mock network but absent from the ToMV/Mock network are indicated with red identification numbers (Fig. 4A), and the genes present in the ToMV/Mock network but absent from the Epl1/Mock network are indicated with blue identification numbers (Fig. 4B). Despite the similarities in the heatmap patterns, there were 89 genes present only in the Epl1/Mock network, whereas nine genes were present only in the ToMV/Mock network (Fig. 4). Moreover, the size of the circle indicates the number of connections a gene has. For instance, MET1 (NBBAC36158) has 13 gene connections in the Ep11/Mock network but it has nine gene connections in the ToMV/Mock network, indicating that METI has more gene connections in plants treated with Epl1. Therefore, the size of the circle in Epl1/Mock is larger than that in ToMV/Mock (Fig. 4). In addition, yellow circles represent the transcription factors (TFs), red circles represent LRR (leucinerich repeat) genes, a blue circle represents a gene involved in the defense response, green circles represent genes involved in epigenetics, and white circles represent genes with unknown functions (Fig. 4). For each gene in the Epl1/Mock or ToMV/Mock networks, the numbers of connected genes are listed in Supplementary Table S2.

Thirteen $T F$ genes were hubs in the Epl1/Mock and ToMV/Mock networks, and each had different numbers of connected genes (Fig. 4). The data indicated that there were more connected genes in Epl1/Mock than in ToMV/Mock. In addition, a GATA TF (NBBAC10673) and an AP2-domain TF (NBBAC42902) were specifically present in the Epl1/Mock network (Fig. 4A). JAZ1 (Jasmonate-zim-domain protein 1, NBBAC99), a GATA TF (NBBAC10673), $U-B O X$ (NBBAC731), TPR (NBBAC7023), and a SOS3IP (NBBAC19532) were specifically present in the Epl1/Mock network (Fig. 4A). Furthermore, JAZ1 was specifically present in the Epl1/Mock network and was connected to a GRAS TF (NBBAC252), which suggests that JA/ET signaling is involved in Epl1-mediated immunity. Interestingly, two groups of genes were connected by EDS1 in the Epl1/Mock network, whereas EDS1 was absent from the ToMV/Mock network (Fig. 4). EDS1 is critical for SAR in plants, suggesting that SA signaling is induced by Epl1 treatment.

Moreover, $7 L R R$ genes were identified in the network analysis, including ZARl (HOPZ-ACTIVATED RESISTANCE 1, NBBAC14246), which has a nucleotide-binding domain and a LRR receptor (NB-LRR). In addition, another $L R R$ gene (NBBAC1092) was specifically present in the Epl1/Mock network (Fig. 4A). In the Epl1/Mock network, 10 of the 21 histone variant genes were specifically identified (Fig. 4A). Furthermore, three histone variant genes (NBBAC34441, NBBAC34443, and NBBAC15044) had more gene connections in the Epl1/Mock network than in the ToMV/Mock network (Fig. 4). Notably, the other specific genes in the Epl1/Mock network have functions that are currently unclear.

\section{DISCUSSION}

\section{T. formosa secretes Epl1}

to trigger viral infection-like immunity.

Similar to vaccine-mediated immunity, the Epl1 elicitor in T. formosa stimulates plant immune responses against bacterial, fungal, and viral pathogens. Several different amino acids in Epl1 and $\mathrm{Sm} 1$ were identified, in this study, based on the differences in each subgroup, providing information for further analyses of Epl1-mediated resistance. Interestingly, the gene expression patterns were similar between the Epl1 and ToMV samples, implying that Epl1 triggers a viral infection-like response. To study Epl1-mediated immunity, the identification of immune response genes, such as LRR receptors, TFs, and immune genes, is fundamental. Because of the similar patterns in the Epl1 and ToMV samples, Epl1-mediated immune genes are difficult to identify by comparing DEGs. We demonstrated that gene-togene networks can highlight several groups of critical genes and provide an explanation for Epl1-mediated plant immunity.

\section{Synergizing JA/ET and SA signaling in Epl1-treated plants.}

In general, there is antagonism between the SA and JA/ET signaling pathways (Brodersen et al. 2006). However, genes present in one or both the SA-JA and SA-ET signaling pathways appear to work synergistically (Norman-Setterblad et al. 2000; Schenk et al. 2000; Xu et al. 1994). Moreover, NPR1 (NONEXPRESSOR OF PR GENE 1) has been shown to cotrigger SAR and ISR and improve disease control (van Wees et al. 2000). $J A Z 1$ and JAR1.2 (JA/ET signaling genes) were up-regulated in the Epl1 sample. JAZ1 was also notable in the Epl1/Mock network. Moreover, EDS1 and NPR1 (SA signaling genes) were also triggered by Epl1, suggesting that synergism between JA/ET and SA signaling occurs in Epl1-treated plants to achieve immunity.

\section{Gene-to-gene network hubs $E D S 1, L R R$ receptor, and histone variant genes.}

In the Epl1/Mock network, two major gene groups were connected by EDS1. EDS1 is an important gene in SAR and eds 1 mutants are susceptible to pathogen infection (Feys et al. 2001). In toll interleukin 1 receptor-nucleotide binding site (NBS)-LRR (TNL) receptor-mediated immunity, EDS1 interacts physically with PAD4 (PHYTOALEXIN DEFICIENT4) to promote the expression of ICS1 (ISOCHORISMATE SYNTHASE1), which results in a feedback loop in which accumulated SA enhances the expression of EDS1 and PAD4 amplifies the resistance (Cui et al. 2017; Vlot et al. 2009). Therefore, EDS1 positively regulates basal immunity against virulent pathogens (Cui et al. 2015). Interestingly, the expression of EDS1 in the Epl1 and ToMV samples showed slight differences. Regarding the gene-to-gene network assay, the threshold was set to DEGs whose expression differed by fourfold. At this threshold, we can see the differences in EDS1 between the Epl1 and ToMV samples. Apart from EDS1, there were 88 Epl1 network-specific genes, and many of these genes also showed slight differences between the two samples. The results of the gene-to-gene network analysis showed that important genes can be distinguished and highlighted, which suggests a direction for additional studies using genetic approaches.

In total, seven LRR genes were notable in the networks, among them, ZARl, which has been well-studied. ZARl is a coiled-coil-NBS-LRR $R$ gene that confers allele-specific recognition of the Pseudomonas syringae HopZ1a effector to trigger resistance (Lewis et al. 2010). Plants can sense earlier encounters with biotic and abiotic stresses through epigenetic regulation, which facilitates genetic responses when stress reappears; this process is referred to as "gene priming." Gene priming can be induced by JA and abscisic acid through chromatin remodeling (histone modification) and epigenetic mark modification (DNA methylation) to superinduce the expression of stress-response genes (Liu and Avramova 2016). Notably, many different histone variants were specifically highlighted in the Epl1/Mock network. These variants can 
further guide exploration of whether gene priming is involved in Epl1-mediated resistance.

In this study, we demonstrated that Epl1 of T. formosa triggers plant immunity against various pathogens. Moreover, we demonstrated how gene-to-gene networks can be used to identify candidate genes for additional studies of Epl1mediated immunity. Most candidate genes are still unclassified and have unknown functions. Gain-of-function and loss-offunction analyses will provide solutions for studying candidate gene functions. However, cloning genes and generating transgenic plants are time consuming and have limited abilities to explore the functions of these genes. The question of how to efficiently evaluate these genes becomes a critical issue and is the next goal for high-throughput genetic investigation.

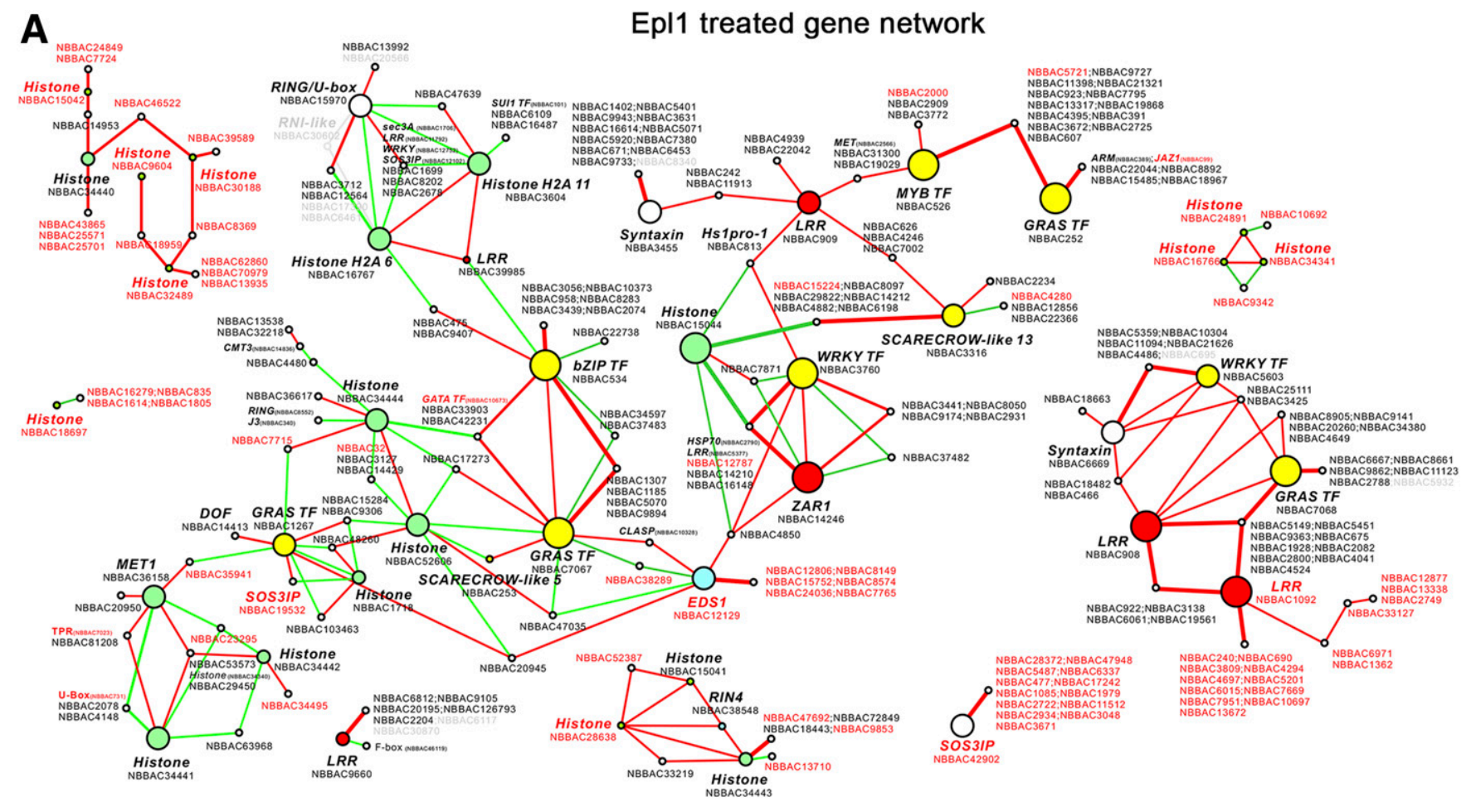

B

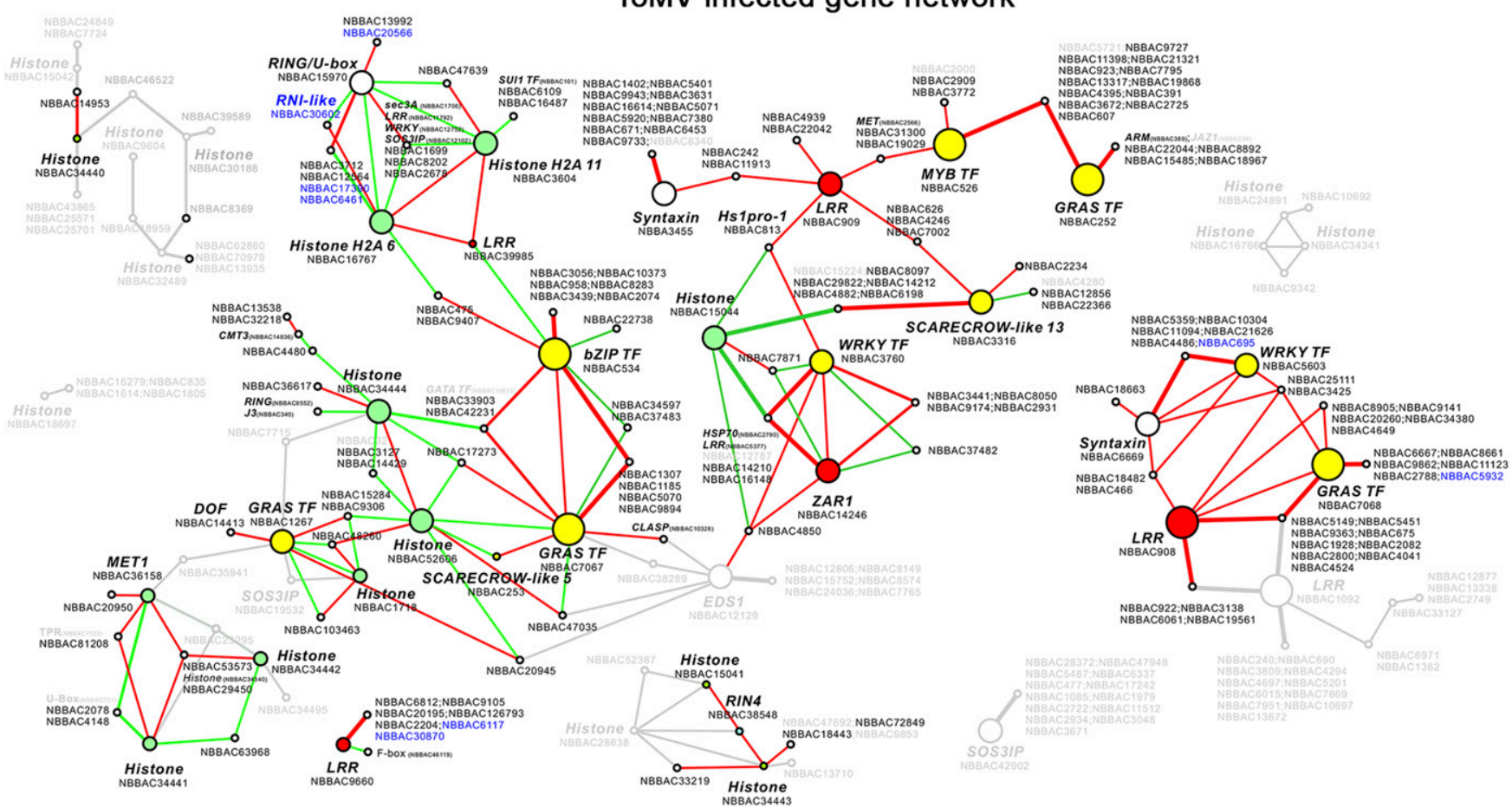

Fig. 4. Gene-to-gene networks. A, Epl1-Mock network (Epl1-treated plants compared with mock-inoculated plants). B, Tomato mosaic virus (ToMV)-Mock network. The red identification numbers indicate the genes specific to panel A, whereas the blue identification numbers indicate the genes specific to panel B. A gray identification number in a given panel indicates that the gene is absent from the other panel. The size of the circle indicates the number of gene connections. Yellow circles represent transcription factor genes; red circles represent leucine-rich repeat (LRR) genes; the blue circle represents defense response gene; green circles represent genes involved in epigenetic regulation; white circles represent genes of unknown function. 


\section{MATERIALS AND METHODS}

\section{Cultivation of $T$. formosa.}

T. formosa was cultivated following the method described by Yang et al. (2009). In brief, T. formosa was grown on potato dextrose agar plates at $25^{\circ} \mathrm{C}$ for 7 days. The conidia of T. formosa (approximately $10^{6}$ per milliliter) were collected by washing with $3 \mathrm{ml}$ of sterile water and inoculating in $250 \mathrm{ml}$ of minimal medium, which was prepared, according to the methods proposed by Cheng et al. (2012), at $25^{\circ} \mathrm{C}$ and shaking at $180 \mathrm{rpm}$ for 7 days.

\section{Purification of Epl1.}

The T. formosa culture medium was centrifuged at $6,000 \times g$ for $20 \mathrm{~min}$. Then, the supernatant was treated with $70 \%$ ammonium sulfate (final concentration $5.5 \mathrm{M}$ ) and was stirred overnight, at $4^{\circ} \mathrm{C}$, for protein precipitation. The precipitated proteins were collected by centrifugation at $12,000 \times g$ for $30 \mathrm{~min}$ at $4^{\circ} \mathrm{C}$ and were resuspended in an extraction buffer $(50 \mathrm{mM}$ Tris- $\mathrm{HCl}$ buffer, $100 \mathrm{mM} \mathrm{NaCl}, \mathrm{pH}$ 7.5). The protein samples were dialyzed in ice-cold extraction buffer, using a Spectra/Por dialysis membrane (molecular weight cutoff 1,000) (Spectrum Laboratories) for $24 \mathrm{~h}$ and the water was changed every $2 \mathrm{~h}$. Then, Epl 1 was purified using ÄKTA FPLC, with a HiPrep16/60 Sephacryl $\mathrm{S}-100 \mathrm{HR}$ column, at a $0.5 \mathrm{ml} / \mathrm{min}$ flow rate. The eluted protein fraction was analyzed by $15 \%$ (wt/vol) sodium dodecyl sulfatePAGE and was stained with Coomassie Blue G250.

\section{LC-MS/MS identification.}

The purified peptides were desalted, using C18 ZipTip (Millipore), prior to LC-MS/MS analyses. The peptides were fractionated using a C18 microcapillary column $(75 \mu \mathrm{m}$ i.d. $\times$ $15 \mathrm{~cm}$ ) with a nano-HPLC system (LC Packings). Reversedphase separation was performed using a linear acetonitrile gradient from $100 \%$ buffer A (5\% acetonitrile to $0.1 \%$ formic acid) to $60 \%$ buffer B ( $80 \%$ acetonitrile to $0.1 \%$ formic acid), for $40 \mathrm{~min}$, with an UltiMate micropump at a flow rate of approximately $200 \mathrm{nl} / \mathrm{min}$. The LC system was coupled to an ion trap mass spectrometer (LCQ DECA XP Plus, ThermoFinnigan) equipped with an electrospray ionization source. Each cycle of one full-scan mass spectrum $(\mathrm{m} / \mathrm{z}, 450$ to 2,000) was followed by three data-dependent tandem mass spectra with the collision energy set at $35 \%$. The peak list was used to query the National Center for Biotechnology Information nonredundant (133,366,630 sequences) database, using the MASCOT program with the following parameters: peptide mass tolerance, $1 \mathrm{Da}$; MS/MS ion mass tolerance, $1 \mathrm{Da}$; enzyme set as trypsin and allowance up to two missed cleavages; variable modifications considering methionine oxidation, cysteine carbamidomethylation, and deamidation of asparagine and glutamine; peptide charge, $2+$ and $3+$; taxonomy limited to fungi (843,964 sequences).

\section{Epl1 treatment and evaluation of resistance.}

For Epl1 treatment, the leaves of $N$. benthamiana and B. rapa subsp. chinensis were sprayed with or without $10 \mathrm{nM}$ Epl1 for 3 days, after which the leaves were inoculated with ToMV and A. brassicicola (approximately $10^{5} \mathrm{CFU} / \mathrm{ml}$ ), respectively. Moreover, $1 \mathrm{nM}$ Epl1 adsorbed onto a paper disk (diameter $8 \mathrm{~mm}$, thickness $1 \mathrm{~mm}$ ) was applied to a carrot (diameter 3 to $4 \mathrm{~cm}$, thickness $5 \mathrm{~mm}$ ) for 3 days; the carrot was then inoculated with P. carotovorum (approximately $10^{6} \mathrm{CFU} / \mathrm{ml}$ ).

The lesions were photographed, and the areas of the lesions were measured at the center of the carrots after 3 days and on $B$. rapa chinensis and $N$. benthamiana after 6 days. The percentage of disease area was calculated using the following formula:

$$
\frac{A_{(c)}-A_{(t)}}{A_{(c)}} \times 100 \%
$$

where $A_{(C)}$ is the leaf area of the plant and $A_{(t)}$ is the nondiseased (spotted) area of the plant. The percentage of plant disease incidence was calculated using the following formula:

$$
\frac{D N}{N} \times 100 \%
$$

where $D N$ is the number of diseased plants and $N$ is the total number of plants.

\section{Total RNA extraction and whole-transcriptome deep sequencing.}

For the T. formosa transcriptome, total RNA was extracted from $1 \mathrm{mg}$ of culture cells with an RNeasy plant mini kit (Qiagen). For the $N$. benthamiana transcriptome, total RNA samples were extracted from Mock, ToMV, Epl1, and Epl1+ToMV leaf tissue, using TRIzol reagent (Invitrogen) according to the manufacturer's protocol. One sample from each condition was used for the NGS data. The whole-transcriptome profiles of the T. formosa and $N$. benthamiana plants were analyzed using an Illumina HiSeq 2000 (Genomics BioSci \& Tech Co.).

\section{Transcriptome and phylogenetic tree analysis.}

The transcriptome expression profiles were analyzed and the database was constructed using the ContigViews web server. The name calling and ORF annotations were determined using the rule-based predictor in ContigViews. Blast2GO software (Blast2GO Co.) was used to analyze the GC and KEGG (Kyoto Encyclopedia of Genes and Genomes) pathways and the domains of the annotated genes. For the phylogenetic tree analysis, the RPB2 nucleotide sequences of Trichoderma spp., including $T$. formosa, were selected for the neighbor-joining analysis with 1,000 bootstrap replicates, using MEGA v7 (Kumar et al. 2016).

\section{Gene-to-gene network analysis.}

To generate the Epl1/Mock network, a list of genes with a greater than fourfold change in expression levels between the Mock and Epl1 samples was identified for the network analysis. Similarly, a list of genes with greater than fourfold changes in expression levels between Mock and ToMV samples was also identified for further analysis. The FPKM values were $\log _{2}$-transformed twice to ensure normality. Genes with transformed FPKM values less than 1.14 were discarded. The final trimmed gene lists for both conditions were used to generate the gene-to-gene networks, using the ContigViews-Network platform. The cutoff values for positive and negative correlations between the genes were set to 0.999 and -0.999 , respectively.

\section{LITERATURE CITED}

Bassel, G. W., Lan, H., Glaab, E., Gibbs, D. J., Gerjets, T., Krasnogor, N., Bonner, A. J., Holdsworth, M. J., and Provart, N. J. 2011. Genome-wide network model capturing seed germination reveals coordinated regulation of plant cellular phase transitions. Proc. Natl. Acad. Sci. U.S.A. 108: 9709-9714.

Brodersen, P., Petersen, M., Bjørn Nielsen, H., Zhu, S., Newman, M. A., Shokat, K. M., Rietz, S., Parker, J., and Mundy, J. 2006. Arabidopsis MAP kinase 4 regulates salicylic acid- and jasmonic acid/ethylenedependent responses via EDS1 and PAD4. Plant J. 47:532-546.

Castro, J. C., Maddox, J. D., Cobos, M., Requena, D., Zimic, M., Bombarely, A., Imán, S. A., Cerdeira, L. A., and Medina, A. E. 2015. De novo assembly and functional annotation of Myrciaria dubia fruit transcriptome reveals multiple metabolic pathways for L-ascorbic acid biosynthesis. BMC Genomics 16:997.

Chan, E. K., Rowe, H. C., Corwin, J. A., Joseph, B., and Kliebenstein, D. J. 2011. Combining genome-wide association mapping and transcriptional 
networks to identify novel genes controlling glucosinolates in Arabidopsis thaliana. PLoS Biol. 9:e1001125.

Cheng, C. H., Yang, C. A., and Peng, K. C. 2012. Antagonism of Trichoderma harzianum ETS 323 on Botrytis cinerea mycelium in culture conditions. Phytopathology 102:1054-1063.

Childs, K. L., Davidson, R. M., and Buell, C. R. 2011. Gene coexpression network analysis as a source of functional annotation for rice genes. PLoS One 6:e22196.

Chisholm, S. T., Coaker, G., Day, B., and Staskawicz, B. J. 2006. Hostmicrobe interactions: shaping the evolution of the plant immune response. Cell 124:803-814.

Cui, H., Gobbato, E., Kracher, B., Qiu, J., Bautor, J., and Parker, J. E. 2017. A core function of EDS1 with PAD4 is to protect the salicylic acid defense sector in Arabidopsis immunity. New Phytol. 213:1802-1817.

Cui, H., Tsuda, K., and Parker, J. E. 2015. Effector-triggered immunity: from pathogen perception to robust defense. Annu. Rev. Plant Biol. 66: 487-511.

Durrant, W. E., and Dong, X. 2004. Systemic acquired resistance. Annu. Rev. Phytopathol. 42:185-209.

Feys, B. J., Moisan, L. J., Newman, M. A., and Parker, J. E. 2001. Direct interaction between the Arabidopsis disease resistance signaling proteins, EDS1 and PAD4. EMBO J. 20:5400-5411.

Ficklin, S. P., and Feltus, F. A. 2011. Gene coexpression network alignment and conservation of gene modules between two grass species: maize and rice. Plant Physiol. 156:1244-1256.

Gao, B., Zhang, D., Li, X., Yang, H., and Wood, A. J. 2014. De novo assembly and characterization of the transcriptome in the desiccationtolerant moss Syntrichia caninervis. BMC Res. Notes 7:490.

Jiménez-Gómez, J. M., Wallace, A. D., and Maloof, J. N. 2010. Network analysis identifies ELF3 as a QTL for the shade avoidance response in Arabidopsis. PLoS Genet. 6:e1001100.

Kim, H. A., Lim, C. J., Kim, S., Choe, J. K., Jo, S. H., Baek, N., and Kwon, S. Y. 2014. High-throughput sequencing and de novo assembly of Brassica oleracea var. Capitata L. for transcriptome analysis. PLoS One 9:e92087.

Kottb, M., Gigolashvili, T., Großkinsky, D. K., and Piechulla, B. 2015. Trichoderma volatiles effecting Arabidopsis: from inhibition to protection against phytopathogenic fungi. Front. Microbiol. 6:995.

Kumar, S., Stecher, G., and Tamura, K. 2016. MEGA7: Molecular Evolutionary Genetics Analysis Version 7.0 for Bigger Datasets. Mol. Biol. Evol. 33:1870-1874.

Lewis, J. D., Wu, R., Guttman, D. S., and Desveaux, D. 2010. Allelespecific virulence attenuation of the Pseudomonas syringae HopZ1a type III effector via the Arabidopsis ZAR1 resistance protein. PLoS Genet. 6:e1000894.

Lin, P. C., Hu, W. C., Lee, S. C., Chen, Y. L., Lee, C. Y., Chen, Y. R., Liu, L. Y., Chen, P. Y., Lin, S. S., and Chang, Y. C. 2015. Application of an integrated omics approach for identifying host proteins that interact with Odontoglossum ringspot virus capsid protein. Mol. Plant-Microbe Interact 28:711-726.

Liu, L. Y., Tseng, H. I., Lin, C. P., Lin, Y. Y., Huang, Y. H., Huang, C. K., Chang, T. H., and Lin, S. S. 2014. High-throughput transcriptome analysis of the leafy flower transition of Catharanthus roseus induced by peanut witches'-broom phytoplasma infection. Plant Cell Physiol. 55: 942-957.

Liu, N., and Avramova, Z. 2016. Molecular mechanism of the priming by jasmonic acid of specific dehydration stress response genes in Arabidopsis. Epigenetics Chromatin 9:8.

Liu, Z. P. 2015. Reverse engineering of genome-wide gene regulatory networks from gene expression data. Curr. Genomics 16:3-22.

Martínez-Medina, A., Fernández, I., Sánchez-Guzmán, M. J., Jung, S. C., Pascual, J. A., and Pozo, M. J. 2013. Deciphering the hormonal signalling network behind the systemic resistance induced by Trichoderma harzianum in tomato. Front. Plant Sci. 4:206.
Mutwil, M., Klie, S., Tohge, T., Giorgi, F. M., Wilkins, O., Campbell, M. M., Fernie, A. R., Usadel, B., Nikoloski, Z., and Persson, S. 2011. PlaNet: combined sequence and expression comparisons across plant networks derived from seven species. Plant Cell 23:895-910.

Mutwil, M., Usadel, B., Schütte, M., Loraine, A., Ebenhöh, O., and Persson, S. 2010. Assembly of an interactive correlation network for the Arabidopsis genome using a novel heuristic clustering algorithm. Plant Physiol. 152:29-43.

Norman-Setterblad, C., Vidal, S., and Palva, E. T. 2000. Interacting signal pathways control defense gene expression in Arabidopsis in response to cell wall-degrading enzymes from Erwinia carotovora. Mol. PlantMicrobe Interact 13:430-438.

Ransbotyn, V., Yeger-Lotem, E., Basha, O., Acuna, T., Verduyn, C., Gordon, M., Chalifa-Caspi, V., Hannah, M. A., and Barak, S. 2015. A combination of gene expression ranking and co-expression network analysis increases discovery rate in large-scale mutant screens for nove Arabidopsis thaliana abiotic stress genes. Plant Biotechnol. J. 13: 501-513.

Salas-Marina, M. A., Isordia-Jasso, M. I., Islas-Osuna, M. A., DelgadoSánchez, P., Jiménez-Bremont, J. F., Rodríguez-Kessler, M., RosalesSaavedra, M. T., Herrera-Estrella, A., and Casas-Flores, S. 2015. The Epl1 and Sm1 proteins from Trichoderma atroviride and Trichoderma virens differentially modulate systemic disease resistance against different life style pathogens in Solanum lycopersicum. Front. Plant Sci. 6:77.

Schenk, P. M., Kazan, K., Wilson, I., Anderson, J. P., Richmond, T., Somerville, S. C., and Manners, J. M. 2000. Coordinated plant defense responses in Arabidopsis revealed by microarray analysis. Proc. Natl Acad. Sci. U.S.A. 97:11655-11660.

Seidl, V., Marchetti, M., Schandl, R., Allmaier, G., and Kubicek, C. P. 2006. Epl1, the major secreted protein of Hypocrea atroviridis on glucose, is a member of a strongly conserved protein family comprising plant defense response elicitors. FEBS J. 273:4346-4359.

Serin, E. A., Nijveen, H., Hilhorst, H. W., and Ligterink, W. 2016. Learning from co-expression networks: possibilities and challenges. Front. Plan Sci. 7:444.

Ton, J., Van Pelt, J. A., Van Loon, L. C., and Pieterse, C. M. 2002. Differential effectiveness of salicylate-dependent and jasmonate/ethylenedependent induced resistance in Arabidopsis. Mol. Plant-Microbe Interact 15:27-34.

van Wees, S. C., de Swart, E. A., van Pelt, J. A., van Loon, L. C., and Pieterse, C. M. 2000. Enhancement of induced disease resistance by simultaneous activation of salicylate- and jasmonate-dependent defense pathways in Arabidopsis thaliana. Proc. Natl. Acad. Sci. U.S.A. 97: 8711-8716.

Vandepoele, K., Quimbaya, M., Casneuf, T., De Veylder, L., and Van de Peer, Y. 2009. Unraveling transcriptional control in Arabidopsis using cis-regulatory elements and coexpression networks. Plant Physiol. 150: 535-546.

Vlot, A. C., Dempsey, D. A., and Klessig, D. F. 2009. Salicylic acid, a multifaceted hormone to combat disease. Annu. Rev. Phytopathol. 47 177-206.

Xu, Y., Chang, P., Liu, D., Narasimhan, M. L., Raghothama, K. G., Hasegawa, P. M., and Bressan, R. A. 1994. Plant defense genes are synergistically induced by ethylene and methyl jasmonate. Plant Cell 6: 1077-1085.

Yang, H. H., Yang, S. L., Peng, K. C., Lo, C. T., and Liu, S. Y. 2009. Induced proteome of Trichoderma harzianum by Botrytis cinerea. Mycol. Res. 113 924-932.

\section{AUTHOR-RECOMMENDED INTERNET RESOURCE}

National Taiwan University's ContigViews web server: www.contigviews.bioagri.ntu.edu.tw 